Open Access

\title{
Low density lipoprotein cholesterol control status among Canadians at risk for cardiovascular disease: findings from the Canadian Primary Care Sentinel Surveillance Network Database
}

\author{
Erfan Aref-Eshghi', Jason Leung ${ }^{1}$, Marshall Godwin ${ }^{1}$, Pauline Duke ${ }^{1}$, Tyler Williamson², Masoud Mahdavian ${ }^{3}$ \\ and Shabnam Asghari ${ }^{1,4^{*}}$
}

\begin{abstract}
Background: To determine the prevalence of uncontrolled LDL-C in patients with high cardiovascular disease (CVD) risks across Canada and to examine its related factors.

Methods: Non-pregnant adults $>20$ years-old, who had a lipid test completed between January 1, 2009 and December 31, 2011 and were included in the Canadian Primary Care Surveillance Network (CPCSSN) database were studied. The Framingham-Risk-Score was calculated to determine the risk levels. A serum $L D L-C$ level of $>2.0 \mathrm{mmol} / \mathrm{L}$ was considered as being poorly controlled. Patients with a previous record of a cerebrovascular accident, peripheral artery disease, or an ischemic heart disease were regarded as those under secondary prevention. Logistic regression modeling was performed to examine the factors associated with the LDL-C control.
\end{abstract}

Results: A total of 6,405 high-risk patients were included in the study and, of this population, $68 \%$ had a suboptimal LDL-C, which was significantly associated with the female gender (OR: 3.26; $95 \% \mathrm{Cl}: 2.63-4.05, p<0.0001$ ) and no medication therapy (OR: 6.31, $95 \%$ Cl: $5.21-7.65, p<0.0001)$. Those with comorbidities of diabetes, hypertension, obesity, and smokers had a better LDL-C control. Rural residents (OR: 0.64, $95 \% \mathrm{Cl}: 0.52-0.78, p<0.0001$ ), and those under secondary prevention (OR: 0.42; $95 \%$ Cl: $0.35-0.51, p<0.0001$ ), were also more likely to have a better LDL-C control.

Conclusion: A high proportion of high-cardiac risk patients in Canadian primary care settings have suboptimal LDL-C control. A lack of medication therapy appears to be the major contributing factor to this situation.

Keywords: Dyslipidemia, Canada, LDL-C control, CPCSSN

\section{Introduction}

Cardiovascular diseases (CVD) are the leading cause of death worldwide with more than 17.3 million deaths in 2008 according to a WHO report [1]. In Canada, CVD is the main cause of death at $32 \%$ and, after musculoskeletal diseases, has the highest economic burden of

\footnotetext{
* Correspondence: shabnam.asghari@med.mun.ca

${ }^{1}$ Memorial University of Newfoundland, St. John's, Newfoundland and Labrador, Canada

${ }^{4}$ Department of Family Medicine, Faculty of Medicine, Memorial University of Newfoundland, Primary Healthcare Research Unit, Agnes Cowan Hostel, Room 425, Health Sciences Centre, 300 Prince Philip Drive, St. John's, NL A1B 3V6, Canada

Full list of author information is available at the end of the article
}

disease [2]. Hyperlipidemia, defined as abnormal blood lipid levels including elevated total cholesterol, lowdensity lipoprotein cholesterol (LDL-C), triglycerides and decreased high-density lipoprotein cholesterol, is well established as a major risk factor to CVD [3]. Similar to many other countries, the management of dyslipidemia, in order to reduce the CVD risk in a clinical setting in Canada, is conducted following the instructions by the available guidelines, including those by the Canadian Cardiovascular Society [4]. These guidelines are modeled after the Framingham Risk Assessment and target specific lipid goals based on the cardiovascular 
risk. Overall, patients with a higher cardiovascular risk are treated more aggressively than those with a lower risk. Thus, patients with a 10-year cardiovascular risk of $>20 \%$ will have an LDL-C goal of $\leq 2 \mathrm{mmol} / \mathrm{L}$, while patients with a 10 -year cardiovascular risk of $<5 \%$ may or may not be treated depending on their initial LDL-C levels. The LDL-C reduction is primarily administered by medication therapy, although diet and physical activity have also been suggested to be effective [5]. Statins are the most commonly used medications for lowering LDL-C levels. Every $1.0 \mathrm{mmol} / \mathrm{L}$ reduction in LDL-C by statins has been reported to be associated with a 23\% relative risk reduction in major vascular events over five years of treatment and follow-up [6]. A $2.0 \mathrm{mmol} / \mathrm{L} \mathrm{ab-}$ solute reduction, or a $50 \%$ relative reduction in LDL-C, provides optimal benefits in CVD risk management; thus, those with a higher CVD risk are more likely to benefit from the management of dyslipidemia [4]. Lipid level targets, however, are sometimes challenging to achieve. While various reports suggest that LDL-C levels are not lowered to target a significant portion of the high-risk populations [6-8], there is no recent report available on the status of LDL-C control among Canadian patients at risk. Canadian guidelines for the diagnosis and treatment of dyslipidemia [4] recommend an LDL-C target goal of $\leq 2.0 \mathrm{mmol} / \mathrm{L}$ for individuals with a high 10 year risk of a cardiovascular event. In the present study, we determine the prevalence of suboptimal LDL-C (LDL-C > $2 \mathrm{mmol} / \mathrm{L}$ ) in high-risk individuals in a primary care setting in Canada. As well, we examine the factors associated with the rates of LDL-C control.

\section{Results}

Among the 22,101 individuals who met the inclusion criteria (Fig. 1), 6,405 (29\%) were classified as high risk, and were included in the analysis (aged 71.9 $\pm 10.3,23 \%$ female, $24 \%$ rural residence). Almost half of the population (46\%) was classified as non-medication users, whereas $12 \%$ and $41.5 \%$ were previous and current users, respectively. The prevalence of those with uncontrolled LDL-C was $68 \%$ and $29 \%$ were determined to be in need of secondary prevention (Fig. 1).

As seen in Table 1, the prevalence of uncontrolled LDL-C gradually decreases with age. High-risk females have a higher rate of uncontrolled LDL-C (77 \%) compared to high-risk males with $66 \%$. Whereas $89 \%$ of non-medication users have sub-optimal LDL-C, the rates are much lower to about $50 \%$ only among previous and current medication users. The rates are approximately $30 \%$ higher among those under primary prevention than those individuals under secondary prevention. Diabetic high-risk patients, however, had a $30 \%$ lower rate of uncontrolled LDL compared to non-diabetics (Table 1).

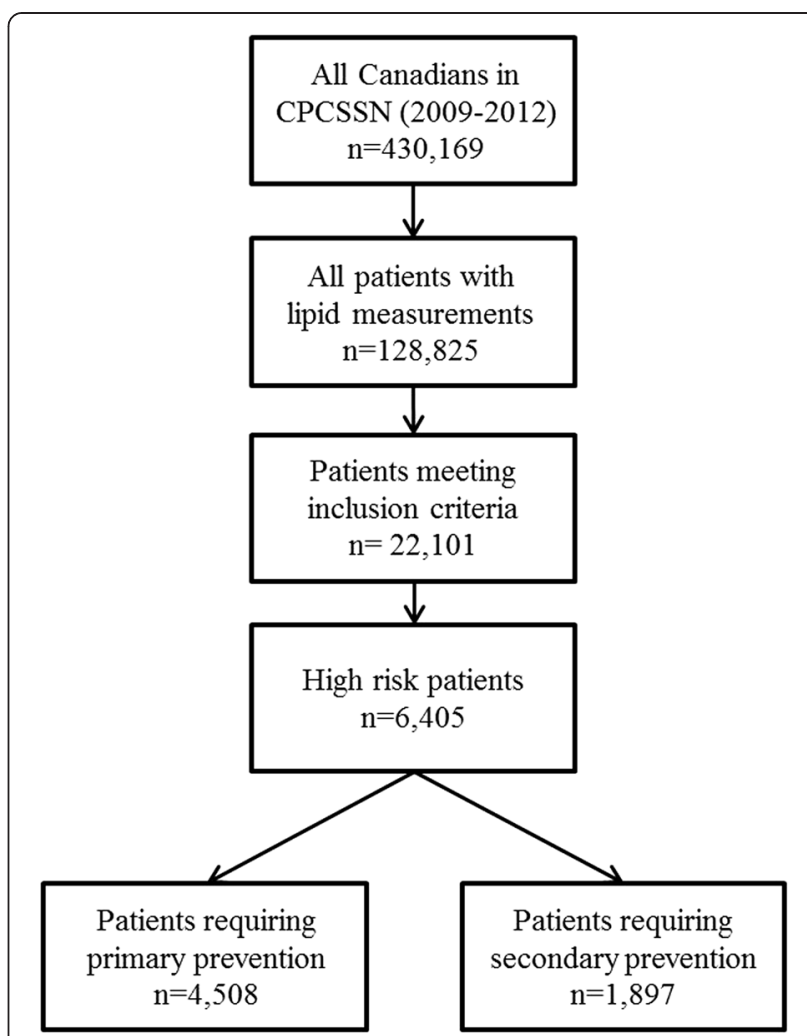

Fig. 1 The flowchart of the study

Table 2 represents the charachteristics of the study population among those with uncontrolled and controlled levels of LDL-C. Table 3 shows the results of the logistic regression model in the high-risk population, adjusting for gender, age, place of residence, comorbidities, and type of prevention. As observed, women are more likely to have an LDL-C above $2.0 \mathrm{mmol} / \mathrm{L}$ than men (OR: 3.26; 95 \% CI: 2.63-4.05, $p<0.0001$ ), and aging is significantly associated with the lower odds of having an uncontrolled LDL-C (OR: 0.95, 95 \% CI: 0.94-0.96, $p<0.0001)$. Comorbidities were associated with a lower rate of suboptimal LDL-C (obesity [OR: 0.62, $95 \%$ CI: 0.46$0.84, p=0.002$ ], current smokers [OR: 0.44, $95 \%$ CI: 0.33$0.59, p<0.0001$ ], diabetes [OR: 0.13, 95 \% CI: 0.11-0.16, $p<0.0001$ ], and hypertension [OR: 0.72, $95 \% \mathrm{CI}: 0.61-$ $0.86, p<0.0001])$. Individuals who were not receiving any lipid-lowering medication had a 6.31 (95 \% CI: 5.21-7.65) fold increased odds of having an LDL-C above $2 \mathrm{mmol} / \mathrm{L}$ compared to current medication users $(p<0.0001)$. Rural residents had a better LDL-C control (OR: 0.64, 95 \% CI: $0.52-0.78, p<0.0001)$ compared to urban dwellers. Patients who were being treated for the purpose of secondary prevention were better controlled in terms of LDL-C (OR: 0.42; 95 \% CI: 0.35-0.51, $p<0.0001)$ than patients who were being treated for the purpose of primary prevention. 
Table 1 Prevalence of uncontrolled LDL among high-risk patients in Canadian EMR primary care settings by category of risk factors

\begin{tabular}{|c|c|c|}
\hline \multirow[t]{2}{*}{ Risk factors } & \multicolumn{2}{|l|}{$\underline{\text { High-risk patients }}$} \\
\hline & Total $(n=6405)$ & Uncontrolled LDL (68.6\%) \\
\hline \multicolumn{3}{|l|}{ Gender } \\
\hline Male & 4931 & $66.0 \%$ \\
\hline Female & 1474 & $77.3 \%$ \\
\hline \multicolumn{3}{|l|}{ Age } \\
\hline$<40$ & 2 & $100.0 \%$ \\
\hline $40-50$ & 72 & $85.7 \%$ \\
\hline $50-60$ & 599 & $81.2 \%$ \\
\hline $60-70$ & 1726 & $72.5 \%$ \\
\hline $70-80$ & 2185 & $66.3 \%$ \\
\hline$>80$ & 1821 & $63.0 \%$ \\
\hline \multicolumn{3}{|l|}{ Smoking status } \\
\hline Current smokers & 1396 & $73.5 \%$ \\
\hline Past smokers & 2850 & $64.1 \%$ \\
\hline Non-smokers & 2159 & $71.4 \%$ \\
\hline \multicolumn{3}{|l|}{ Diabetes } \\
\hline Diabetics & 2715 & $51.6 \%$ \\
\hline Non-diabetics & 3690 & $81.0 \%$ \\
\hline \multicolumn{3}{|l|}{ Hypertension } \\
\hline Hypertensive & 3911 & $66.9 \%$ \\
\hline Non-hypertensive & 2494 & $71.2 \%$ \\
\hline \multicolumn{3}{|l|}{ Obesity } \\
\hline Normal/underweight & 1218 & $70.5 \%$ \\
\hline Overweight & 2754 & $70.3 \%$ \\
\hline Obese & 2433 & $61.1 \%$ \\
\hline \multicolumn{3}{|c|}{ Lipid-lowering medication use } \\
\hline Non-users & 2958 & $89.5 \%$ \\
\hline Previous users & 789 & $50.4 \%$ \\
\hline Current users & 2658 & $50.6 \%$ \\
\hline \multicolumn{3}{|l|}{ Place of residence } \\
\hline Rural & 4754 & $70.5 \%$ \\
\hline Urban & 1501 & $67.8 \%$ \\
\hline \multicolumn{3}{|l|}{ Type of prevention } \\
\hline Primary & 4508 & $77.3 \%$ \\
\hline Secondary & 1897 & $47.9 \%$ \\
\hline
\end{tabular}

\section{Discussion}

Our study is the first of its kind to assess the status of LDL-C control in a targeted population for CVD primary and secondary prevention in a primary care setting across Canada. We used a cross-sectional study design using the electronic medical records of the Canadian population under primary care that is at a high risk for CVD. The information for risk calculation was only available for less than a quarter of the subjects who had
Table 2 CVD associated factors among high-risk patients with and without controlled levels of LDL-C in Canadian EMR primary care settings

\begin{tabular}{llll}
\hline Risk factors & Controlled LDL & Uncontrolled LDL & $P$-value \\
\hline Age (Mean \pm SD) & $73.80 \pm 9.57$ & $71.27 \pm 10.4$ & $<0.0001$ \\
Gender (female) & $17.5 \%$ & $26.5 \%$ & $<0.0001$ \\
$\begin{array}{l}\text { Body mass index } \\
\text { (Mean } \pm \text { SD) }\end{array}$ & $29.93 \pm 5.84$ & $28.85 \pm 5.39$ & $<0.0001$ \\
Diabetes & $65 \%$ & $32 \%$ & $<0.0001$ \\
Hypertension & $64 \%$ & $59 \%$ & $<0.0001$ \\
Smoking & & & \\
$\quad$ Non-smokers & $30.8 \%$ & $35.2 \%$ & $<0.0001$ \\
$\quad$ Past smokers & $50.9 \%$ & $41.6 \%$ & \\
$\quad$ Current smokers & $18.1 \%$ & $23.1 \%$ & $<0.0001$ \\
Lipid-lowering medication use & & \\
$\quad$ Non-user & $15.5 \%$ & $60.4 \%$ & 0.055 \\
Previous user & $19.3 \%$ & $8.9 \%$ & $<0.0001$ \\
$\quad$ Current user & $65.1 \%$ & $30.6 \%$ & \\
Residence (rural) & $22.3 \%$ & $25.1 \%$ & \\
Prevention (secondary) & $49.00 \%$ & $20.60 \%$ &
\end{tabular}

a record of completing a lipid test; this is mainly due to the missing information on smoking ( $70 \%)$, suggesting that FRS is not measured for a large proportion of patients in primary care despite the recommendations by the guidelines. It also indicates that the smoking status, a well-known risk factor of CVD, is not regularly tracked and recorded by family physicians. This raises the question as to what extent the FRS is being practiced and

Table 3 Multivariate logistic regression model for uncontrolled LDL among high risk patients in Canadian EMR primary care settings ${ }^{a}$

\begin{tabular}{|c|c|c|}
\hline Risk factors (reference group) & Odds ratio $(95 \% \mathrm{Cl})$ & $P$-value \\
\hline Gender (males) & $3.26(2.63-4.05)$ & $<0.0001$ \\
\hline Age & $0.95(0.94-0.96)$ & $<0.0001$ \\
\hline \multicolumn{3}{|l|}{ Obesity (BMI $\leq 25)$} \\
\hline Overweight & $1.05(0.77-1.42)$ & 0.775 \\
\hline Obese & $0.62(0.46-0.84)$ & 0.002 \\
\hline \multicolumn{3}{|l|}{ Smoking status (nonsmokers) } \\
\hline Past smokers & $0.85(0.71-1.01)$ & 0.062 \\
\hline Current smokers & $0.44(0.33-0.59)$ & $<0.0001$ \\
\hline Diabetes & $0.13(0.11-0.16)$ & $<0.0001$ \\
\hline Hypertension & $0.72(0.61-0.86)$ & $<0.0001$ \\
\hline \multicolumn{3}{|c|}{ Lipid-lowering medication (current users) } \\
\hline Previous users & $1.28(1.02-1.61)$ & 0.035 \\
\hline Non-users & $6.31(5.21-7.65)$ & $<0.0001$ \\
\hline Place of residence (urban) & $0.64(0.52-0.78)$ & $<0.0001$ \\
\hline Type of prevention (primary) & $0.42(0.35-0.51)$ & $<0.0001$ \\
\hline
\end{tabular}

${ }^{a}$ Patients with diabetes and hypertension were compared to those without diabetes or hypertension, respectively 
whether it is influencing the primary care practice at all, since many practitioners might use other criteria as an indication of LDL-C lowering treatment. Nevertheless, according to our findings, approximately three-quarters of the high-risk population have poorly controlled LDLC. The status of control is better among men, the elderly, rural residents, those under secondary prevention management, those taking lipid modifying agents, and those with comorbidities. In our study, lipid modifying agents have the greatest effect on the status of LDL-C control. Non-medication users have 6.3 fold increased odds of having poorly controlled LDL-C compared to current users. Previous medication users were not significantly different compared with non-users, which suggests that the effect of lipid lowering medications does not endure three months from cessation. Approximately $90 \%$ of the lipid lowering medication users were on statins; similar results were identified where we limited the study to statin users. Therefore, it could be hypothesized that a lack of medication therapy may be a major contributor to a low rate of LDL-C control in Canada, which may possibly be related to a lack of updated knowledge among physicians or the unwillingness of patients to participate in medication therapy. Since half of the current medication users still have suboptimal levels of LDL-C, other factors, such as a lack of physical activity and appropriate dieting among the patients, as well as the satisfaction of physicians with LDL-C levels reaching close to the cut-off point, may also be considered. Moreover, of special importance are the status of nutrition and the use of nutraceutical in treating dyslipidemia, given that several studies report that these agents, in addition to statin therapy, need to be considered in treating dyslipidemia [7]. Since we did not have access to this information, our multivariate model cannot predict the effect of these factors.

Although the number of similar studies performed on LDL-C control worldwide is limited and, to our knowledge, no such report is available from Canada, the findings of this study are comparable to those few studies conducted elsewhere. In a Chinese study, only $37 \%$ of patients with Coronary Heart Disease had a desirable LDL-C level [8]. This figure was lower among very high-risk patients and differed among the two genders and various age groups. In a Brazilian study, only $30 \%$ of the patients obtained the advisable LDL-C levels and the rates were significantly lower among those with poor income [9]. A recent study of 17.5 million statin treated high-risk adults in the United States [10] found that $\sim 30 \%$ of the statintreated patients had not reached the recommended LDL-C levels of $100 \mathrm{mg} / \mathrm{dl}(2.5 \mathrm{mmol} / \mathrm{L})$, and $75 \%$ had an LDL-C $>70 \mathrm{mg} / \mathrm{dl}(1.8 \mathrm{mmol} / \mathrm{L})$ deemed optional according to the American guidelines [11] at the time of the study. These figures would possibly increase if the study had taken into consideration the untreated populations.
The main recommendation by the Canadian guidelines for high-risk patients is to reduce the LDL-C to $\leq 2.0 \mathrm{mmol} / \mathrm{L}$. Alternatively, an $\geq 50 \%$ reduction from the baseline LDL-C, in addition to an apoB target level of less than $0.8 \mathrm{~g} / \mathrm{L}$, can be implemented. It might be possible that some of the health-care providers follow the alternative recommendations based on which the LDL-C levels do not have to be lowered to $2.0 \mathrm{mmol} / \mathrm{L}$. Otherwise, they might have not found the presented evidence by the guidelines compelling and made an informed valued decision not to initiate pharmacologic treatment. These inconsistencies may account for a portion of those with uncontrolled LDL-C, a subject matter that is not possible to assess merely from electronic medical records, since we did not have access to the baseline levels of lipids at the time the therapy was initiated for the patients.

This study is limited by several factors; i.e., the high risk population was defined according the Canadian Cardiovascular Guidelines at the time of the study (2009) [4]. Recent cardiovascular guidelines still consider FRS $\geq 20 \%$ as a high risk of CVD; however, other disease conditions, e.g., patients with chronic kidney disease have been added to the high-risk groups and recommended for cardiovascular risk management. The patients in our study are not treated and evaluated according to the new guidelines and thus their status of the newly added high-risk medical conditions is not known.

Among the 128,825 individuals who had lipid testing during the study period, 22,101 $(<20 \%)$ individuals had a measure of cardiovascular risk factors within six months of the lipid tests. In order to avoid the possibility of bias due to the unavailable information, we compared the LDL-C levels of those with and without risk classification data. We observed a minimal difference between the two groups suggesting a low possibility of selection bias (uncontrolled LDL-C: 80 vs. $83 \%$, LDL-C levels: 2.91 vs. $2.87 \mathrm{mmol} / \mathrm{dl}$, respectively).

The cross-sectional nature of the study did not accurately permit the interpretation of the causal and temporal relationships across the variables. As such, the relationship between LDL-C control and its associated factors, including CVD, diabetes and hypertension, should be interpreted with caution in terms of the therapeutic regime suggested by the CVD prevention guidelines for patients with those conditions [4]. Since a similar pattern is observed for aging, obesity and smoking, the latter explanation seems more likely as all of the patients in our study were high risk and most likely under more stringent therapeutic measures. These factors are indicators of higher Framingham-Risk-Scores and it is more likely to draw the attention of the practitioners to such treatments. As well, it was not possible to represent the variability of lipid and risk levels over time, given that they change constantly. We did not have 
information on medication dosage to determine if the medication users were on adequate dosage; however, the study findings show that lipid-lowering medication users were more likely to attain the lipid goals compared to non-users. There was no information available from the family histories of the patients on the comorbidities, such as Type II Diabetes, which is known to increase the risk of CVDs in the non-diabetes population [12, 13]. Finally, the prevention class categorization may not be ideal, given that the database included information from the year 2006 onward. In that regard, the patients would still be classified into primary prevention, despite the possibility of a disease condition CVED or IHD occurrence before 2006, even though previous studies show that physicians keep updated EMR profiles of those patients at risk of cardiovascular diseases [14].

\section{Conclusion}

A large portion of high-risk patients in Canada have suboptimal LDL-C control. A lack of medication therapy seems to be the most potential contributing factor to this condition, which could result from alternative recommendations or informed decisions by the health-care providers. Our data suggests that there is significant opportunity for improvement. Further studies may also be needed to elucidate other potential causes of uncontrolled LDL-C in Canada, specifically variations in medical practice and patients' adherence and tolerance. In addition, providers in the health-care system need to identify the barriers to medication adherence and implement new methods to improve medication adherence in high-risk patients.

\section{Methods}

\section{Study design}

This is a cross-sectional study using a secondary analysis of the data from the Canadian Primary Care Sentinel Surveillance Network (CPCSSN) database.

\section{Data source}

CPCSSN is Canada's first library of digital information based on point-of-care data from primary-care practices. Data from these EMRs is extracted quarterly and uploaded in a de-identified format to both regional and central (pan-Canadian) databases. The database contains a comprehensive record of all diagnoses, health conditions, risk factors, laboratory tests, medication use, as well as other services provided by family physicians in Canada. It is increasingly used for chronic disease surveillance in primary care and also as a tool for conducting primary-care research [15]. At the time of this study, the CPCSSN database included data from close to 600 primary-care clinicians in rural and urban settings across ten Canadian provinces with data on 844,592 individuals [16].

\section{Study population}

The study population included adults at high risk of CVD according to Canadian guidelines at the time of the study [4]. According to these guidelines, the patients with a 10 -year risk $\geq 20 \%$ of developing CVD were considered as high risks and were included in the study.

To identify the high-risk patients, all non-pregnant adults aged 20 years and older who had a lipid profile in their record between January 1, 2009 and December 31, 2011 were identified from the CPCSSN database. The 10 -year risk score of CVD was calculated using the risk factors measured within six months of the lipid tests according to the Framingham Risk Assessment (FRS) chart for a 10-year risk of a total CVD event [4].

Variables required for CVD risk estimation, including comorbidities, level of blood lipids, level of systolic blood pressure, smoking status and medical history were extracted from the CPCSSN database. The most recent information regarding the lipid levels and the variables required for CVD risk estimation from every patient, was used in the event that the patient was visited multiple times during the study period.

\section{Study variables}

LDL-C control is the primary outcome variable in this study. High-risk patients with a serum LDL-C level $\geq 2.0 \mathrm{mmol} / \mathrm{L}$ were considered as being poorly controlled, whereas those with lower LDL-C levels were regarded as being well controlled.

Independent variables, including age at the time of the lipid test, gender, place of residence, body mass index, smoking status, medical history of diabetes, hypertension and lipid lowering medication use were extracted from the database. Diabetes and hypertension were defined using CPCSSN algorithms for chronic conditions [17] which have high sensitivity, specificity and positive predictive value to detect these conditions with all scores being over $80-90 \%[18,19]$. Obesity was defined as BMI $\geq 30$; whereas those with BMI lower than 30 , but higher than 25 , were classified as overweight. The smoking status was extracted from the most recent record by the family physician at the time of the lipid test, and individuals were classified as non-smokers, past smokers, and current smokers. Place of residence (rural/urban) was determined using the first two characters of the postal code from each individual.

Lipid lowering medication (HMG-CoA reductase inhibitors, Fibrates, Bile Acid Sequestrants, Nicotinic Acid, and other agents) usage was classified into three categories as follows: 1) current user was defined as lipid-lowering medication use at the time of the lab test (started before the date of blood test and did not stop up to three months before the time of the blood test); 2) previous user was defined as the existence of any record of lipid-lowering 
medication use within two years from the date of the lab test and a record of discontinuation of at least three months before the date of the lab test; and 3) non-user was defined as no record of medication use within two years of the lab test. The type of lipid lowering medications was not specified in the analysis since more than $90 \%$ of the medication users were on statins.

Patients with any record (diagnostic text, ICD code) of previous Ischemic Heart Disease (IHD), Peripheral Vascular Disease (PVD), or Cerebrovascular Disease (CVD) were categorized as those under secondary prevention. All other patients with no record of these conditions in the same period were regarded as those in need of primary prevention.

\section{Statistical analysis}

Characteristics of the study population, as well as the mean and confidence intervals of the individual lipid components, were summarized using descriptive statistics. Classical tests of hypothesis, including the student's $T$-test and the chi-squared test, were conducted to test for the association between variables. Logistic regression modeling was used to examine the association between LDL-C control status and possible influencing factors. Variable age was treated continuously. A $p$-value of less than 0.05 was considered statistically significant. All analyses were performed using STATA/SE 11.2 (Stata Corp., College Station, Texas, USA).

\section{Ethics}

The study protocol was approved for ethics by the Health Research Ethics Authority (HREA) of Newfoundland and Labrador. Patients' records and information were anonymous and de-identified prior to the analysis.

\section{Competing interests}

The authors declare that they have no competing interests.

\begin{abstract}
Authors' contributions
Study design: SA, JL EA, MG; Data collection: SA, TW; Statistical analysis: SA, EA, JL; Interpretation of the results: SA, EA, MG, PD, TW, MM; Manuscript writing: SA, EA, JL; Critical comments on the manuscript: MG, PD, TW, MM. All authors read and approved the final manuscript.
\end{abstract}

\section{Funding source}

The study was not supported by any funding source.

\footnotetext{
Author details

${ }^{1}$ Memorial University of Newfoundland, St. John's, Newfoundland and Labrador, Canada. ${ }^{2}$ University of Calgary, Calgary, Alberta, Canada. ${ }^{3}$ University of Ottawa, Ottawa, Ontario, Canada. ${ }^{4}$ Department of Family Medicine, Faculty of Medicine, Memorial University of Newfoundland, Primary Healthcare Research Unit, Agnes Cowan Hostel, Room 425, Health Sciences Centre, 300 Prince Philip Drive, St. John's, NL A1B 3V6, Canada.
}

Received: 11 May 2015 Accepted: 10 June 2015

Published online: 24 June 2015

\section{References}

1. World Health Organization. Global status report on noncommunicable diseases 2010. 2011. Available from: http://www.who.int/nmh/publications/ ncd report full_en.pdf last accessed April 15th 2015.

2. Public Health Agency of Canada, Canadian Institute for Health Information, Heart and Stroke Foundation of Canada, Statistics Canada. Tracking heart disease and stroke in Canada. 2009. Available from: http://www.phacaspc.gc.ca/publicat/2009/cvd-avc/pdf/cvd-avs-2009-eng.pdf last accessed March 15th 2015.

3. Musunuru K. Atherogenic dyslipidemia: cardiovascular risk and dietary intervention. Lipids. 2010;45(10):907-14

4. Genest J, McPherson R, Frohlich J, Anderson T, Campbell N, Carpentier A, et al. 2009 Canadian Cardiovascular Society/Canadian guidelines for the diagnosis and treatment of dyslipidemia and prevention of cardiovascular disease in the adult-2009 recommendations. Can J Cardiol. 2009;25(10):567-79.

5. Leon AS, Sanchez OA. Response of blood lipids to exercise training alone or combined with dietary intervention. Med Sci Sports Exerc. 2001;33(6 Suppl):S502-15. Discussion S528-9.

6. Baigent C, Keech A, Kearney PM, Blackwell L, Buck G, Pollicino C, et al. Efficacy and safety of cholesterol-lowering treatment: prospective meta-analysis of data from 90,056 participants in 14 randomized trials of statins. Lancet. 2005;366(9493):1267-78.

7. Scicchitanoa P, Camelib M, Maielloc M, Modestid PA, Muiesane ML, Novo S, et al. J Funct Foods. 2014;6:11-32.

8. Ding RJ, Ma CS, Chen H, Wu Y, Yang XC, Hua Q, et al. Control rate of increased low-density lipoprotein cholesterol levels in cardiology outpatients with coronary heart disease in Beijing. Zhonghua Xin Xue Guan Bing Za Zhi. 2013;41(3):251-5.

9. Vacanti LJ, Santos SC, Fujita AM, Lima DS, Lopes AF, Vetorazi R, et al. Low rate of achieving LDL-cholesterol objective in a low income population. Arq Bras Cardiol. 2005;85(3):162-5.

10. Jones PH, Nair R, Thakker KM. Prevalence of dyslipidemia and lipid goal attainment in statin-treated subjects from 3 data sources: a retrospective analysis. J Am Heart Assoc. 2012;1, e001800.

11. Jellinger PS, Smith DA, Mehta AE, Ganda O, Handelsman Y, Rodbard HW, et al. American Association of Clinical Endocrinologists' guidelines for management of dyslipidemia and prevention of atherosclerosis. Endocr Pract. 2012;18 Suppl 1:1-78.

12. Pannacciulli N, De Pergola G, Ciccone M, Rizzon P, Giorgino F, Giorgino R. Effect of family history of type 2 diabetes on the intima-media thickness of the common carotid artery in normal-weight, overweight, and obese glucose-tolerant young adults. Diabetes Care. 2003;26(4):1230-4.

13. Ciccone MM, Scicchitano P, Cameli M, Cecere A, Cortese F, Dentamaro I. Endothelial function in pre-diabetes. Diabetes and diabetic cardiomyopathy: a review. J Diabetes Metab. 2014;5:364. doi:10.4172/2155-6156.1000364.

14. Ivers N, Pylypenko B, Tu K. Identifying patients with ischemic heart disease in an electronic medical record. J Prim Care Community Health. 2011;2(1):49-53.

15. Asghari S, Aref-Eshghi E, Hurley O, Godwin M, Duke P, Williamson T, et al. Does the prevalence of dyslipidemias differ between Newfoundland and the rest of Canada? Findings from the Electronic Medical Records of the Canadian Primary Care Sentinel Surveillance Network. Front Cardiovasc Med. 2015;2:1.

16. Birtwhistle R, Keshavjee K, Lambert-Lanning A, Godwin M, Greiver M, Manca $D$, et al. Building a pan-Canadian primary care sentinel surveillance network: initial development and moving forward. J Am Board Fam Med. 2009:22(4):412-22

17. Kadhim-Saleh A, Green M, Williamson T, Hunter D, Birtwhistle R. Validation of the diagnostic algorithms for 5 chronic conditions in the Canadian Primary Care Sentinel Surveillance Network (CPCSSN): a Kingston Practice-based Research Network (PBRN) report. J Am Board Fam Med. 2013;26(2):159-67.

18. Coleman N, Halas G, Peeler W, Casaclang N, Williamson T, Katz A. From patient care to research: a validation study examining the factors contributing to data quality in a primary care electronic medical record database. BMC Fam Pract. 2015;16(1):11.

19. Williamson T, Green ME, Birtwhistle R, Khan S, Garies S, Wong ST, et al. Validating the 8 CPCSSN case definitions for chronic disease surveillance in a primary care database of electronic health records. Ann Fam Med. 2014;12(4):367-72. 